\title{
On the gravitational radiation from the collapse of neutron stars to rotating black holes
}

\author{
Luca Baiotti $^{1}$, Ian Hawke ${ }^{2}$ and Luciano Rezzolla ${ }^{1,3}$ \\ ${ }^{1}$ Max-Planck-Institut für Gravitationsphysik, Albert-Einstein-Institut, 14476 Golm, Germany \\ 2 School of Mathematics, University of Southampton, Southampton SO17 1BJ, UK \\ ${ }^{3}$ Department of Physics, Louisiana State University, Baton Rouge, LA 70803, USA
}

Received 21 December 2006, in final form 15 February 2007

Published 30 May 2007

Online at stacks.iop.org/CQG/24/S187

\begin{abstract}
We provide details and present additional results on the numerical study of the gravitational-wave emission from the collapse of neutron stars to rotating black holes in three dimensions. More specifically, we concentrate on the advantages and disadvantages of the use of the excision technique and on how alternative approaches to that of excision can be successfully employed. Furthermore, as a first step towards source characterization, we present a systematic discussion of the influence that rotation and different perturbations have on the waveforms and hence on the energy emitted in gravitational waves.
\end{abstract}

PACS numbers: 04.25.Dm, 04.30.Db, 04.70.Bw, 95.30.Lz, 97.60.Jd

\section{Introduction}

The study of the gravitational collapse of rotating stars to black holes is a cornerstone of any theory of gravity and a long-standing problem in general relativity. Important issues in relativistic astrophysics awaiting clarification, such as the mechanism responsible for $\gamma$-ray bursts, may be unveiled with a more detailed understanding of the physics of gravitational collapse in rotating and magnetized stars. Furthermore, the study of gravitational collapse will provide the waveforms and the energetics of one of the most important sources of gravitational radiation.

In our previous work [1, 2], we have described how we can perform accurate threedimensional relativistic simulations of such events and how we are able to extract their gravitational-wave signals. Before our work, the only work in the literature about the gravitational radiation from neutron-star collapse dates back 20 years and is restricted to axisymmetry [3]. Here, after a brief introduction to our code and to the models we have simulated, we give more details on the techniques and results presented in $[1,2]$, focussing on the properties of the gravitational waves produced and on how these are influenced by factors such as rate of rotation of the compact star or the type and amplitude of the perturbations introduced to trigger the collapse. 
Throughout the paper we use a spacelike signature $(-,+,+,+)$ and a system of units in which $c=G=M_{\odot}=1$ (unless explicitly shown otherwise for convenience). Greek indices are taken to run from 0 to 3 , Latin indices from 1 to 3 and we adopt the standard convention for the summation over repeated indices.

\section{Basic equations and their implementation}

The Whisky code solves the general-relativistic hydrodynamics equations on a threedimensional numerical grid with Cartesian coordinates [4]. The code has been constructed within the framework of the Cactus Computational Toolkit (see [5, 6] for details), and it is developed at the Albert Einstein Institute and at the Louisiana State University. This public domain code provides high-level facilities such as parallelization, input/output, portability on different platforms and several evolution schemes to solve general systems of partial differential equations. Clearly, special attention is dedicated to the solution of the Einstein equations, whose matter-terms in non-vacuum spacetimes are handled by the Whisky code.

In other words, while the Cactus code provides at each time step and on a spatial hypersurface the solution of the Einstein equations

$$
G_{\mu \nu}=8 \pi T_{\mu \nu},
$$

where $G_{\mu \nu}$ is the Einstein tensor and $T_{\mu \nu}$ is the stress-energy tensor, the Whisky code provides the time evolution of the hydrodynamics equations, expressed through the conservation equations for the stress-energy tensor $T^{\mu \nu}$ and for the matter current density $J^{\mu}$

$$
\nabla_{\mu} T^{\mu \nu}=0, \quad \nabla_{\mu} J^{\mu}=0 .
$$

In what follows, and mostly for the sake of completeness, we give a brief overview of how both the right- and the left-hand sides of equations (1) are computed within the coupled Cactus/Whisky codes. The equations presented have already been discussed in several different publications, e.g. in $[1,7,8]$, and we refer the interested readers to these works for more details.

\subsection{Evolution of the field equations}

Many different formulations of the equations have been proposed throughout the years, starting with the ADM formulation in 1962 [9]. As mentioned in the introduction, we use the NOK [10] formulation, which is based on the ADM construction and has been further developed in [11].

Details of our particular implementation of the conformal traceless reformulation of the ADM system as proposed by [10-12] are extensively described in [7, 13] and will not be repeated here. We only mention, however, that this formulation makes use of a conformal decomposition of the 3-metric, $\tilde{\gamma}_{i j}=\mathrm{e}^{-4 \phi} \gamma_{i j}$, and the trace-free part of the extrinsic curvature, $A_{i j}=K_{i j}-\gamma_{i j} K / 3$, with the conformal factor $\phi$ chosen to satisfy $\mathrm{e}^{4 \phi}=\gamma^{1 / 3}$, where $\gamma$ is the determinant of the spatial 3-metric $\gamma_{i j}$. In this formulation, in addition to the evolution equations for the conformal 3-metric $\tilde{\gamma}_{i j}$ and the conformal traceless extrinsic curvature $\tilde{A}_{i j}$, there are evolution equations for the conformal factor $\phi$, for the trace of the extrinsic curvature $K$ and for the 'conformal connection functions' $\tilde{\Gamma}^{i} \equiv \tilde{\gamma}^{i j}{ }_{, j}$. We note that although the final mixed, first- and second-order, evolution system for the variables $\left\{\phi, K, \tilde{\gamma}_{i j}, \tilde{A}_{i j}, \tilde{\Gamma}^{i}\right\}$ is not in any immediate sense hyperbolic, there is evidence showing that the formulation is at least equivalent to a hyperbolic system [14-16]. In the formulation of [11], the auxiliary variables $\tilde{F}_{i}=-\sum_{j} \tilde{\gamma}_{i j, j}$ were used instead of the $\tilde{\Gamma}^{i}$. 
2.1.1. Gauge choices. The code is designed to handle arbitrary shift and lapse conditions, which can be chosen as appropriate for a given spacetime simulation. More information about the possible families of spacetime slicings which have been tested and used with the present code can be found in $[7,17]$. Here, we limit ourselves to recalling details about the specific foliations used in the present evolutions. In particular, we have used hyperbolic $K$-driver slicing conditions of the form

$$
\partial_{t} \alpha=-f(\alpha) \alpha^{2}\left(K-K_{0}\right),
$$

with $f(\alpha)>0$ and $K_{0} \equiv K(t=0)$. This is a generalization of many well-known slicing conditions. For example, setting $f=1$ we recover the 'harmonic' slicing condition, while, by setting $f=q / \alpha$, with $q$ an integer, we recover the generalized ' $1+\log$ ' slicing condition [18]. In particular, all the simulations discussed in this paper are done using condition (3) with $f=2 / \alpha$. This choice has been made mostly because of its computational efficiency, but we are aware that 'gauge pathologies' could develop with the ' $1+\log$ ' slicings $[19,20]$.

For the spatial gauge, we use one of the 'Gamma-driver' shift conditions proposed in [17] (see also [13]), that essentially act so as to drive the $\tilde{\Gamma}^{i}$ to be constant. In this respect, the 'Gamma-driver' shift conditions are similar to the 'Gamma-freezing' condition $\partial_{t} \tilde{\Gamma}^{k}=0$, which, in turn, is closely related to the well-known minimal distortion shift condition [21]. The differences between these two conditions involve the Christoffel symbols and are basically due to the fact that the minimal distortion condition is covariant, while the Gamma-freezing condition is not.

In particular, all the results reported here have been obtained using the hyperbolic Gammadriver condition,

$$
\partial_{t}^{2} \beta^{i}=F \partial_{t} \tilde{\Gamma}^{i}-\eta \partial_{t} \beta^{i},
$$

where $F$ and $\eta$ are, in general, positive functions of space and time. For the hyperbolic Gamma-driver conditions it is crucial to add a dissipation term with coefficient $\eta$ to avoid strong oscillations in the shift. Experience has shown that by tuning the value of this dissipation coefficient it is possible to almost freeze the evolution of the system at late times. We typically choose $F=3 / 4$ and $\eta=3$ and do not vary them in time.

The singularity-avoiding properties of the above gauge choices have proved equally good both when using excision, as we did in [8] and [1], and when not using excision. In this latter case, the addition of a small amount of dissipation in the metric and gauge terms is necessary to obtain long-term stable evolutions [2]. In the absence of an excised region of spacetime, the gauge choices (4) are essential to 'freeze' the evolution in those regions of the computational domain inside the apparent horizon, where the metric functions experience the growth of very large gradients.

\subsection{Evolution of the hydrodynamics equations}

An important feature of the Whisky code is the implementation of a conservative formulation of the hydrodynamics equations [22-24], in which the set of equations (2) is written in a hyperbolic, first-order and flux-conservative form of the type

$$
\partial_{t} \mathbf{q}+\partial_{i} \mathbf{f}^{(i)}(\mathbf{q})=\mathbf{s}(\mathbf{q})
$$

where $\mathbf{f}^{(i)}(\mathbf{q})$ and $\mathbf{s}(\mathbf{q})$ are the flux-vectors and source terms, respectively [25]. Note that the right-hand side (the source terms) does not depend on derivatives of the stress-energy tensor. Furthermore, while the system (5) is not strictly hyperbolic, strong hyperbolicity is recovered in a flat spacetime, where $\mathbf{s}(\mathbf{q})=0$. 
Additional details of the formulation we use for the hydrodynamics equations can be found in [25]. We stress that an important feature of this formulation is that it allows for the extension to a general relativistic context of the powerful numerical methods developed in classical hydrodynamics, in particular high-resolution shock-capturing schemes based on exact [26-28] or approximate Riemann solvers (see [25] for a detailed bibliography). Such schemes are essential for a correct representation of shocks, whose presence is expected in several astrophysical scenarios.

For all the results presented here, we have solved the hydrodynamics equations employing the Marquina flux formula and a third-order PPM [29] reconstruction, and the Einstein field equations using a Runge-Kutta scheme of third order, the ' $1+\log$ ' slicing condition and the 'Gamma-driver' shift conditions [17]. After having seen no significant difference in the dynamics of our models while using polytropic or ideal-fluid EOSs (because no shocks form), we have concentrated only on the former, which require slightly less computational time.

\subsection{Mesh refinement}

An important improvement with respect to the work presented in [8], which we refer to as paper I hereafter, is the possibility of solving now both the fields and hydrodynamics equations on non-uniform grids using a 'box-in-box' mesh refinement strategy [30] (see figure 2 of [31]). All the simulations of paper I were redone with the new grid setup and no differences with respect to the unigrid results were found as far as the dynamics of the matter and of the horizons are concerned. On the other hand, this change introduces two important advantages: firstly, it reduces the influence of inaccurate boundary conditions at the outer boundaries which can be moved far from the central source; secondly, it allows for the wave zone to be included in the computational domain and thus for the extraction of important information about the gravitational-wave emission produced during the collapse.

In practice, we have adopted a Berger-Oliger prescription for the refinement of meshes on different levels [32] and used the numerical infrastructure described in [30], i.e., the Carpet mesh refinement driver for Cactus (see [33] for details). In addition to this, in [1] we had also used a simplified form of adaptivity in which new refined levels are added at predefined positions during the evolution. This progressive mesh refinement, which allows us to use much less computational resources, was the key improvement to our previous code [8] and allowed us to extract, for the first time in three-dimensional calculations, the gravitational waveform from the collapse to a rotating black hole. While a fixed or a progressive mesh-refinement technique leads to no appreciable change in the dynamics of the matter or of the horizons, it can influence the spectral distribution of the radiation emitted especially at high frequencies [31]. Unless explicitly stated otherwise, all the results presented here have been computed using seven fixed levels of refinements, the coarsest of which having a grid spacing $h / M_{\odot}=2.56$ (the grid spacing of each finer nested grid is one half of the grid spacing of the containing grid) and extending to a distance $d / M_{\odot}=307.2$ from the centre. We want to stress that on any currently available machine it would be impossible to perform simulations on a uniform grid, with boundaries so far out to include the wave zone and inner resolutions high enough to accurately resolve the star and the horizon (see also section 5).

\subsection{Singularity excision}

The use of the excision technique was essential in paper I for studying the dynamics of the collapse with uniform grids, because these, combined with the computational resources available at that time, had forced us to use a relatively coarse resolution (and outer boundaries 
close to the stellar surface). Such a resolution was sufficient to describe accurately the dynamics of the matter and of the horizons but also required the use of excision if the simulation was to be carried out beyond horizon formation.

An alternative to the use of the excision technique consists in adding a small amount of dissipation to the evolution equations for the metric and gauge variables and in relying on the use of singularity-avoiding gauges and of high resolution to extend the simulations well past the formation of the apparent horizon. More specifically, we have used an artificial dissipation of the Kreiss-Oliger type [34] on the right-hand sides of the evolution equations for the spacetime variables and the gauge quantities. This is needed mostly because all the field variables develop very steep gradients in the region inside the apparent horizon. Under these conditions, small high-frequency oscillations (either produced by finite-differencing errors or by small reflections across the refinement or outer boundaries) can easily be amplified, leave the region inside the apparent horizon and rapidly destroy the solution. In practice, for any time-evolved quantity $u$, the right-hand side of the corresponding evolution equation is modified with the introduction of a term of the type $\mathcal{L}_{\text {diss }}(u)=-\varepsilon h^{3} \partial_{i}^{4} u$, where $h$ is the grid spacing, and $\varepsilon$ is the dissipation coefficient, which is allowed to vary in space.

We have experimented with configurations in which the coefficient was either constant over the whole domain or larger for the grid points inside the apparent horizon. We noted no significant difference between these two cases. Much more sensitive is instead the choice of the value of $\varepsilon$. In the simulations reported here, the employed values of $\varepsilon$ are between 0.0075 and 0.02 . For each initial model, two values of $\varepsilon, \varepsilon_{\min }$ and $\varepsilon_{\max }$, can be determined, such that for values smaller than $\varepsilon_{\min }$ the dissipation is not strong enough to cure the instability, and such that for values larger than $\varepsilon_{\max }$ the solution is different from that obtained without dissipation (over-dissipation). Such differences in the solution cannot be seen in the dynamics of the matter or of the horizons, but only in the very sensitive waveforms.

The use of numerical dissipation stops the growth of the metric functions, which, instead of growing more and more while approaching the singularity, stabilize to a stationary state. Outside the horizon, the spacetime is practically identical to that obtained without dissipation and the dynamics of the horizon itself are the same as in the case in which excision was performed (up to when the latter data are available). In contrast, the metric inside the horizon is rather far from being a solution of the Einstein equations, but this does not influence the outside spacetime, as shown also in figure 2 of [2]. Hereafter, all of the presented results will refer to simulations carried out without excision and we note that no dissipation is added to the evolution of any matter variable.

\section{Initial stellar models}

As mentioned earlier, this paper is specially dedicated to the study of the gravitational collapse of slowly and rapidly rotating supramassive relativistic stars, in uniform rotation, that have become unstable to axisymmetric perturbations. Given equilibrium models of gravitational mass $M$ and central energy density $e_{c}$ along a sequence of fixed angular momentum or fixed rest mass, the Friedman, Ipser and Sorkin criterion $\partial M / \partial e_{c}=0$ [35] can be used to locate the exact onset of the secular instability to axisymmetric collapse. The onset of the dynamical instability to collapse is located near that of the secular instability but at somewhat larger central energy densities. Unfortunately, no simple criterion exists to determine this location, but the expectation mentioned above has been confirmed by the simulations performed here and by those discussed in [36]. Note that, in the absence of viscosity or strong magnetic fields, the star is not constrained to rotate uniformly after the onset of the secular instability and could 
Table 1. Equilibrium properties of the initial stellar models. The different columns refer respectively to: the central rest-mass density $\rho_{c}$, the ratio of the polar to equatorial coordinate radii $r_{p} / r_{e}$, the gravitational mass $M$, the circumferential equatorial radius $R_{e}$, the angular velocity $\Omega$, the ratio $J / M^{2}$ where $J$ is the angular momentum, the ratio of rotational kinetic energy to gravitational binding energy $T /|W|$. All models have been computed with a polytropic EOS with $K_{\mathrm{ID}}=100$ and $\Gamma=2$.

\begin{tabular}{llllllll}
\hline Model & $\rho_{c}\left(\times 10^{-3}\right)$ & $r_{p} / r_{e}$ & $M$ & $R_{e}$ & $\Omega\left(\times 10^{-3}\right)$ & $J / M^{2}$ & $T /|W|\left(\times 10^{-2}\right)$ \\
\hline$D 0$ & 3.325 & 1.00 & 1.636 & 7.54 & 0.00 & 0.000 & 0.00 \\
$D 0.5$ & 3.314 & 0.99 & 1.644 & 7.59 & 0.92 & 0.108 & 0.32 \\
$D 1$ & 3.280 & 0.95 & 1.665 & 7.74 & 1.73 & 0.206 & 1.16 \\
$D 1.5$ & 3.249 & 0.91 & 1.690 & 7.91 & 5.76 & 0.281 & 2.13 \\
$D 2$ & 3.189 & 0.85 & 1.728 & 8.21 & 2.88 & 0.362 & 3.52 \\
$D 2.5$ & 3.162 & 0.81 & 1.756 & 8.43 & 3.20 & 0.410 & 4.48 \\
$D 3$ & 3.134 & 0.75 & 1.797 & 8.80 & 3.55 & 0.468 & 5.79 \\
$D 3.5$ & 3.121 & 0.73 & 1.810 & 8.93 & 3.65 & 0.485 & 6.20 \\
$D 4$ & 3.116 & 0.65 & 1.861 & 9.65 & 3.95 & 0.543 & 7.67 \\
\hline
\end{tabular}

develop differential rotation. In realistic neutron stars, however, very intense magnetic fields are likely to counteract this.

For simplicity, we have focused on initial models constructed with a polytropic EOS $p=K \rho^{\Gamma}$, choosing $\Gamma=2$ and polytropic constant $K_{\mathrm{ID}}=100$ to produce stellar models that are, at least qualitatively, representative of what is expected from observations of neutron stars. More specifically, we have selected the models with the following procedure: first we have identified nine models having polar-to-equatorial axes ratio in the interval 0.65-1.0 and lying on the line defining the onset of the secular instability (the dotted line in figure 1). The models used as initial data have then been derived from the secularly unstable ones after increasing the central energy density by $5 \%$, while keeping the same axis ratio. These models were indeed found to be dynamically unstable $[8]$ and we have indicated them here as $D 0, D 1, \ldots, D 3.5, D 4$ following the convention introduced in paper I. Note that model $D 0$ effectively corresponds to a TOV star.

The main properties of these models are summarized in figure 1, which shows the gravitational mass as a function of the central energy density for equilibrium models constructed with the chosen polytropic EOS. The solid, dashed and dotted lines correspond respectively to: the sequence of non-rotating models, the sequence of models rotating at the mass-shedding limit and the sequence of models that are at the onset of the secular instability to axisymmetric perturbations. The dynamically unstable initial models used in the collapse simulations are shown as circles.

A more quantitative description of the models is presented in table 1, which summarizes the main equilibrium properties of the initial models. The circumferential equatorial radius is denoted as $R_{e}$, while $\Omega$ is the angular velocity with respect to an inertial observer at infinity, and $r_{p} / r_{e}$ is the ratio of the polar to equatorial coordinate radii. Other quantities shown are the central rest-mass density $\rho_{c}$, the ratio of the angular momentum $J$ to the square of the gravitational mass $M$, and the ratio of rotational kinetic energy to gravitational binding energy $T /|W|$.

\section{Dynamics of the collapse}

In paper I we have described in detail the dynamics of the matter and of the apparent and event horizons during the gravitational collapse. Here, we summarize previous results and provide additional details and comparisons. 


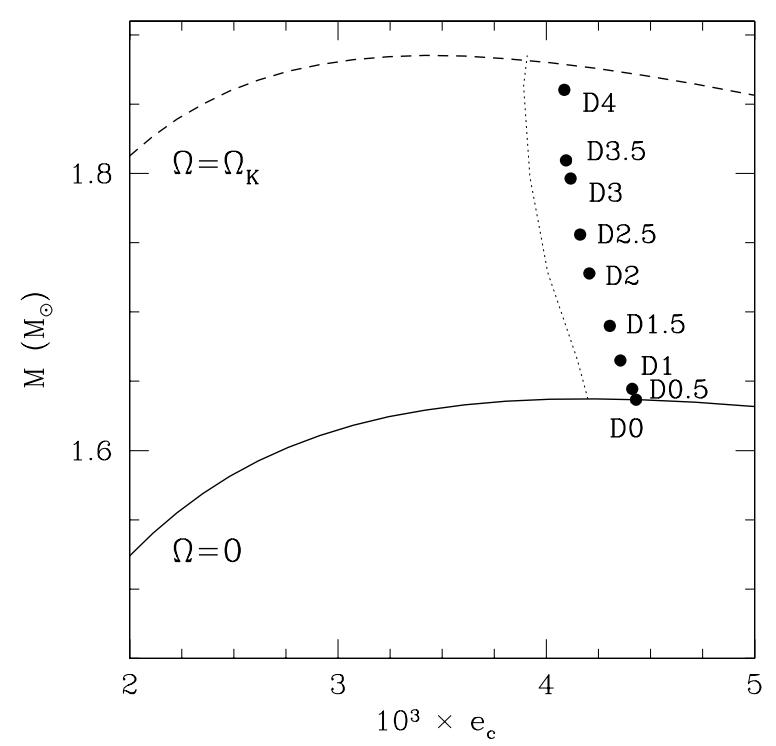

Figure 1. Gravitational mass shown as a function of the central energy density for equilibrium models constructed with the polytropic EOS, for $\Gamma=2$ and polytropic constant $K_{\mathrm{ID}}=100$. The solid, dashed and dotted lines correspond to the sequence of non-rotating models, the sequence of models rotating at the mass-shedding limit and the sequence of models that are at the onset of the secular instability to axisymmetric perturbations. Also shown are the dynamically unstable (filled circles) initial models used in the collapse simulations.

We start by noting that although dynamically unstable models are expected to collapse over a dynamical timescale, the collapse is traditionally accelerated through the introduction of a small perturbation, either in terms of an added radial velocity or through a slight and global reduction of the pressure. This is done, for instance, by using a polytropic constant for the evolution $K$ that is $2 \%$ smaller than the one used to calculate the initial data $K_{\mathrm{ID}}$. We note that we do not solve for the constraint equations once the initial perturbation is introduced. This clearly produces a small error but, as shown in paper I, after an initial transient lasting a couple of tenths of millisecond, the constraint violation differs only a few per cent from the one measured in a simulation in which the constraints had been resolved. A more detailed discussion of the influence of the type and amplitude of the perturbations introduced on the waveforms emitted will be presented in section 6 .

Overall, all of the models, with different initial $J / M^{2}$ values, show similar dynamics as far as the bulk of the matter and the horizons are concerned. The main differences across different models concern, instead, the dynamics of the matter around the equatorial plane and the surface of the star. Of course, models with higher $J / M^{2}$ are initially more flattened and their oblateness increases as the collapse proceeds, leading during the collapse to the temporary formation of a disc-like configuration, which is however unstable and is rapidly accreted (cf figures 5 and 6 of paper I).

As a good representative quantity, we show in the left panel of figure 2 the time evolution of the maximum value of the rest-mass density for some of the models. Clearly all curves show an exponential growth of the type $\rho=\rho_{0}+A \exp \left[\left(t-t_{0}\right) / \tau\right]$, where $\rho_{0}$ and $t_{0}$ refer to the initial values, suggesting that the growth time increases with the rate of rotation, as a result of the increased centrifugal support. This is made more clear in the right panel of figure 2, which shows the exponential growth time $\tau$ as a function of the rotation rate. The open 

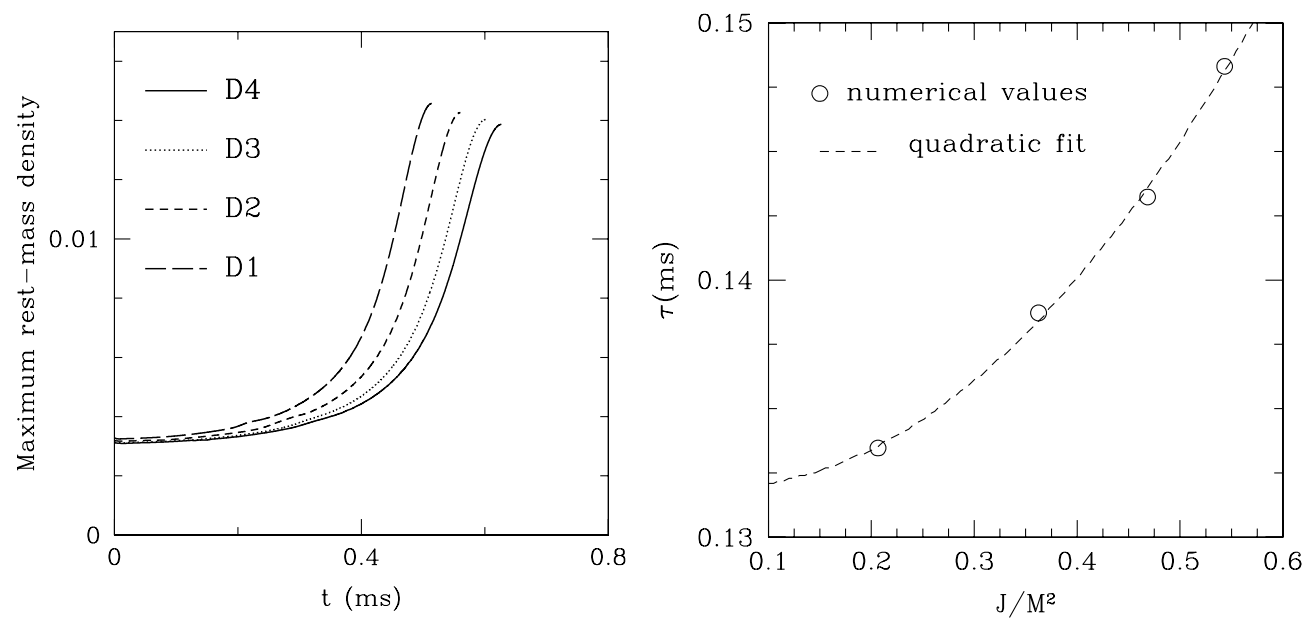

Figure 2. Left panel: time evolution of the maximum value of the rest-mass density for some representative models. Right panel: exponential growth time $\tau$ of the central density as a function of stellar rotation rate $J / M^{2}$. Indicated with open circles are the numerical values, while the dashed line is the very good fit obtained with a quadratic function in $J / M^{2}$.



Figure 3. Time evolution of the mass of the horizon in the isolated/dynamical-horizon framework, for the different models (for clarity's sake, not all models are shown). The dotted lines represent the respective values of the ADM masses at the initial time, as computed after a compactification to infinity.

circles represent the numerical values, while the dashed line is the excellent fit obtained with a quadratic function of $J / M^{2}$ with coefficients $c_{0}=0.13208, c_{1}=-0.00655, c_{2}=0.06642$, where $c_{i}$ is the coefficient of the term of order $\left(J / M^{2}\right)^{i}$. The increase of the growth time with the rotation rate is simple to explain in terms of the increased centrifugal support that rapidly rotating models have and its quantification represents an important result, being the first estimate of the growth time for the dynamical instability to axisymmetric perturbations as computed in full general relativity and for rapidly rotating stars.

The evolution of another representative quantity is presented in figure 3 , which shows the behaviour of the masses of the isolated horizons (see [37-41] and the discussion in paper I) 



Figure 4. Gravitational-wave extraction at short distances: waveforms of the even-parity metric perturbations $Q_{20}^{+}$(left panel) and $Q_{40}^{+}$(right panel) as functions of retarded time (shown both in ms and solar-mass units) for model $D 4$ evolved on a uniform grid. Different lines refer to different extraction distances, expressed in $\mathrm{M}$ in the legend and corresponding, respectively, to coordinate radii 1.6, 2.6 and 3.6 times the initial coordinate stellar equatorial radius $R_{*}$.

compared to the initial values of the ADM masses computed at spatial infinity. As in paper I, the figure shows that the mass of the newly formed black hole is measured very accurately, and with an error, at the resolutions we have used here, of a couple of per cent only, when compared with the expected value of the ADM mass. Furthermore, this error is indeed comparable with the error coming from the use of a finite-size domain and is about one per cent (see paper I for more details).

\section{Extracting in the wave zone}

The simulations presented in paper I, as well as other works (e.g. [36, 42, 43]), made use of numerical grids with uniform spacing. This, together with the presently available computational resources, has initially forced us to place the outer boundary of our computational domain in the near zone, i.e. in regions of the spacetime where the gravitational waves have not yet reached their asymptotic form, which instead happens in what is usually referred to as the wave zone. Under these constraints, the data on the gravitational waveforms that we extract through gauge-invariant perturbative techniques (see [31, 44, 45] for details) do not provide interesting information besides the obvious change in the quadrupole moment of the background spacetime.

This is illustrated in figure 4, where we show the even-parity metric perturbations $Q_{20}^{+}$ (left panel) and $Q_{40}^{+}$(right panel) as functions of retarded time (shown both in ms and solarmass units) extracted at coordinate radii 1.6, 2.6 and 3.6 times the initial coordinate stellar equatorial radius, $R_{*}$, or equivalently at distances $8.1 M, 13.4 M, 18.8 M$. These extraction 2 -spheres are clearly not far enough out to be in the wave zone. Indeed, we see (left panel) that the waveforms for the $\ell=2$ mode compared at the same retarded time do not overlap, as they should if they were computed in the wave zone, since the invariance under a retarded-time scaling is a property of the solutions of a wave equation. The overlapping for the $\ell=4$-mode 
waves (right panel) is slightly better and although quite noisy they show wave-like behaviour. Clearly this is possible because the higher-frequency waves have a shorter wavelength and, less influenced by the secular changes of the metric, reach the wave zone at smaller radii. However, also in this case there clearly are secular variations of the waveforms that are probably related to the dynamics of the gravitational field in the near zone. We also note that the amplitude of the $\ell=4$ mode is much smaller (one or two orders of magnitude) than that of the $\ell=2$ mode, so one has to look primarily at the latter mode to ascertain whether wave extraction has been performed successfully.

Using the mesh-refinement setup discussed in section 2.3, we were able to place the numerical boundary of our coarsest grid much farther out. For our fiducial simulation we use an outer boundary located at $\sim 160 M$ from the central object. Because of the approximate boundary conditions employed, at some time in the simulation numerical errors reflected from the outer boundary arrive back in the central high-gradient zone where they excite numerical instabilities which are not cured by the small amount of dissipation applied. Given the relatively short duration of the collapse, however, this does not represent a serious problem and it is always possible to place the outer boundary far enough out so that its influence is delayed to a time when the largest part of the gravitational-wave emission has already taken place. This boundary distance is indeed around $160 \mathrm{M}$ for all of the models studied here. Outer-boundary locations placed farther out have not produced significant differences in the waveforms nor on the emitted energy. In particular, comparing simulations of model D4 with outer boundary located at $160 \mathrm{M}$ and at $320 \mathrm{M}$, the maximal pointwise relative difference between correspondent values of the $\ell=2$ mode is below $1 \%$ (but the average difference is about $0.1 \%$ ) and the relative difference in the emitted energy is below $0.5 \%$.

Finally, we note that our extraction 2-spheres are not located near the outer boundary but, rather, around $50 \mathrm{M}$ from the origin and thus at a distance which is about four times larger than the gravitational wavelength. This distance is a good compromise between being far enough in the wave zone and far enough from the outer boundary, from where numerical contamination may come. We note that a similar choice (i.e. extraction at 50M) was made in [3].

\section{Variations on the theme: factors influencing the waveforms}

A fundamental prospect of the world-wide effort dedicated to the construction and planning of gravitational-wave detectors is that of opening a 'new window' on the universe through which we may observe details of compact objects which would not be accessible through other astronomical observations. As a step towards gravitational-wave astronomy and the characterization of the sources through the features of their gravitational radiation, we now discuss how the waveforms computed here can provide important information on the physical properties of the collapsing star. More specifically, we do this by considering how the form and amplitude of the waves are influenced by factors such as the rotation rate of the collapsing star or the type and amplitude of the initial perturbations.

\subsection{The role of rotation}

Assessing the role that the stellar rotation rate has on the emitted gravitational radiation is particularly simple in the case of uniformly rotating stars, as all models can be selected so as to differ only in the value of the angular velocity $\Omega$ after having fixed either the central energy density or the gravitational mass. Here, however, we consider the role of rotation along the sequence of dynamically unstable models that we have discussed in the previous sections. More specifically, we show in figure 5 the waveforms computed for some of the simulated 



Figure 5. $Q_{20}^{+}$measured at $\sim 50 M$ in simulations of some of the models $D 0-D 4$, all with an initial pressure depletion of $2 \%$. Filled circles indicate the coordinate retarded times at which the apparent horizon is first found.

models when the initial model has been induced to collapse through a reduction of about $2 \%$ for the initial pressure support. The filled circles in the figure indicate the coordinate retarded times at which the apparent horizon is first found. (This notation differs from that we used in figures 1 and 3 of [2], where we showed with a circle the time of first apparent-horizon appearance in absolute time. This was done to stress how short our simulations with excision extended past the horizon formation.) 
The waveform reported in the upper left panel is at least two orders of magnitude smaller than any other waveform presented in figure 5, because it refers to the non-rotating star $D 0$ and should, at least in principle, be exactly zero. Model $D 0$, however, is not exactly a spherical star but rather a Cartesian approximation of a spherical star at the level of resolution considered here. Hence, the gravitational-wave signal in the upper left panel should not be considered as an intrinsic error but, rather, as a measure of the overall accuracy of our evolution code and extraction technique.

A rapid look at the waveforms in figure 5 is sufficient to realize that the amount of initial rotational velocity does influence both the amplitude and the form of the emitted gravitational radiation. A more detailed discussion of this in terms of the energy efficiency and of the spectral properties of the signal will be presented in section 7. Here, however, it is sufficient to underline that while the form of the signal does not vary considerably, its amplitude changes by more than two orders of magnitude over the range of possible rotations considered.

An interesting feature which is common to all the waveforms reported in figure 5 is the presence of a high-frequency signal between 0 and $0.25 \mathrm{~ms}$ and whose amplitude does not change appreciably with rotation. As we will discuss in the following section, this initial and spurious burst of radiation is most likely the signature of a perturbation in the star which is further amplified by the reduction in pressure. Finally, we note that, in all the considered cases, the complete signal has been collected, starting from the initial spurious burst at the beginning of the collapse and up to the ring-down phase of the black hole. After this, the extracted signal becomes essentially constant until the numerical error produced at the outer boundary reaches the region of the spacetime where the fields are rapidly varying in space and destroys the solution (this is not shown in the figure).

Finally, the different panels of figure 6 offer information about the properties of the waveforms that is complementary to that presented in figure 5. More specifically, they show the power spectral density (PSD) of the waveforms emitted by models $D 3$ and $D 4$ in the absence of any initial perturbation. The left panels, in particular, show the PSD of the complete signal and thus including also the initial spurious burst (see section 6.2 for a discussion of this), while the right panels show the PSD of only the final part of the waveforms, namely the one starting from the retarded time at which the apparent horizon is first found, and that could be used to approximately bracket the ring-down of the black hole. Indicated with a vertical dotted line is the corresponding frequency of the fundamental black-hole quasi-normal mode (QNM) as computed in [46]. As expected, the PSD of the complete signal is rather narrow and shows a main peak around $6 \mathrm{kHz}$ and a series of smaller peaks at larger frequencies, related to the initial burst of radiation and possibly a signature of the $w$ modes of the perturbed star. The PSD of the QNM ringing, on the other hand, is wider in frequency but very well matched with the expected frequency of the fundamental QNM. It is also worth noting that the spectra in the right panels have a rather poor accuracy in frequency, as the timeseries used is intrinsically short.

It is worth remarking that while the stellar models considered here are still quite idealized, they are sufficiently realistic to highlight a precise correlation between the spectral features of the waveforms and the black-hole QNMs. We expect this feature to survive also more realistic treatments of the stellar matter.

\subsection{The role of the pressure perturbation}

As discussed in section 3, it is customary in simulations of collapse to rotating black holes to introduce a pressure perturbation whose amplitude can be rather large (as in [3], where the pressure support was decreased by up to $99 \%$ ) or rather small (as in paper I, where the 



Figure 6. Power spectra of $h_{+}$measured at $\sim 50 M$. All panels refer to initial data without any added perturbation. Top left: complete extracted signal for $D 3$; top right: only ring-down signal for $D 3$; bottom left: complete signal for $D 4$; bottom right: only ring-down signal for $D 4$.

pressure support was decreased by only $2 \%$ ). The rationale behind this approach is that the introduction of the perturbation simply increases the amplitude of the (only) unstable mode, hence triggering the instability and decreasing the computational costs. As we will show below, this assumption is correct only for very small perturbations and, quite to the contrary, large-amplitude perturbations can have a strong impact on both the dynamics of the matter (and hence of the horizons) and the gravitational waveforms.

We start by comparing in figure 7 the evolution of the central rest-mass density for model $D 4$ in the case in which a pressure perturbation of $2 \%$ is introduced (i.e. $\Delta p / p=2 \%$ ) and when no explicit perturbation is introduced (i.e. $\Delta p / p=0 \%$ ). In this latter case, the fact that the model is already past the secular instability limit and the presence of a small but nonzero truncation error are sufficient to trigger the instability, which leads to a collapse (delayed with respect to the perturbed case). The two curves in figure 7 are properly shifted in time so as to be superposed and the upper $x$-axis is used to indicate the coordinate time in the case of the unperturbed collapse. It is evident that in this regime of linear perturbations the dynamics of 


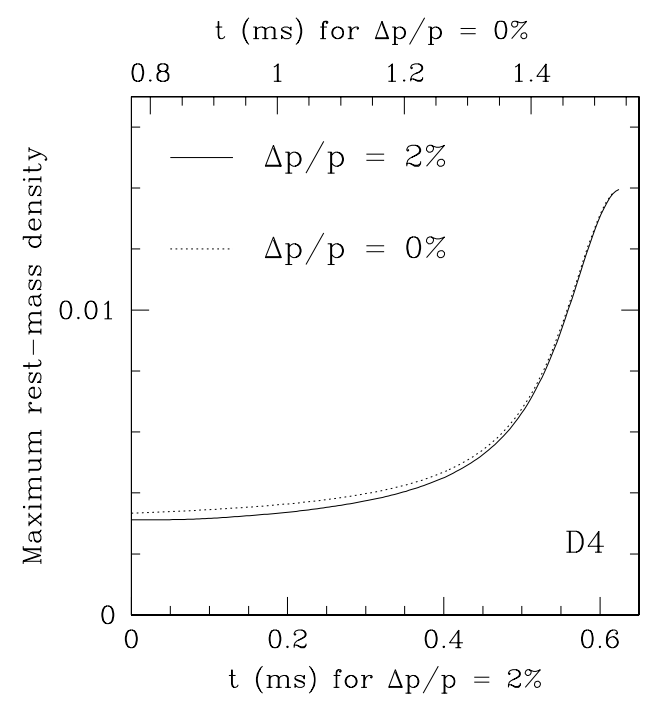

Figure 7. Comparison of the time evolution of the maximum value of the rest-mass density for model $D 4$ with and without pressure depletion at the initial time. The curve for the unperturbed case has been shifted in time to offer a direct comparison. The time reference for the non-perturbed data is reported on the upper axis.
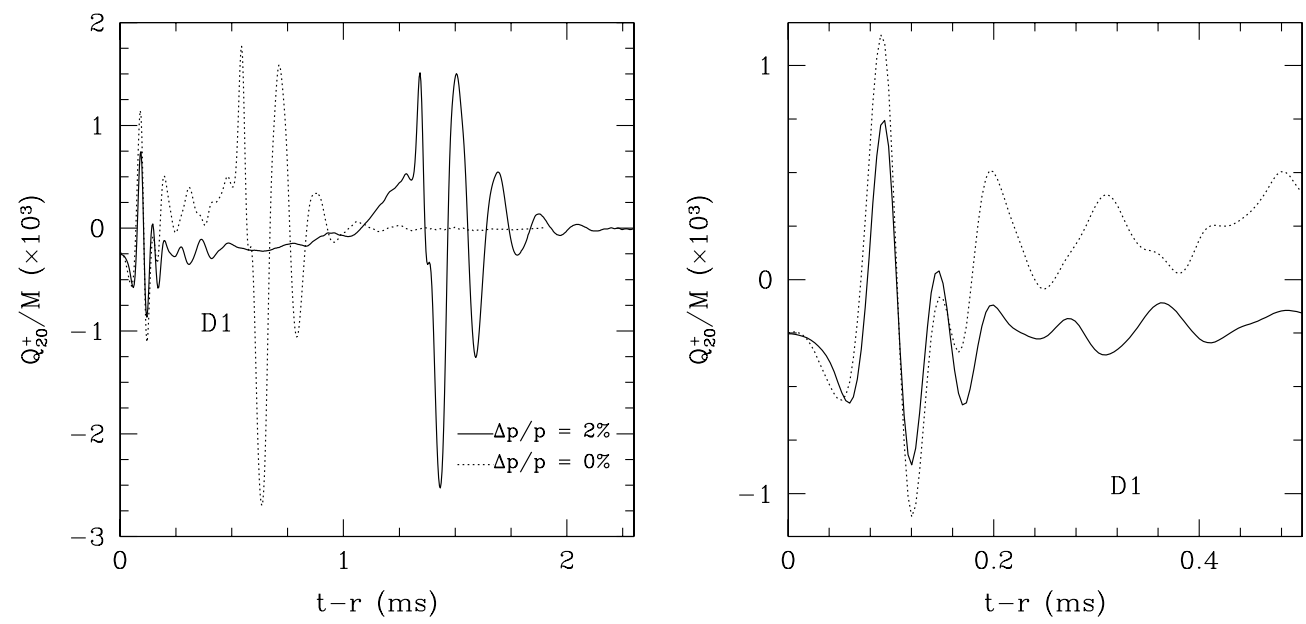

Figure 8. Left panel: comparison of the $Q_{20}^{+}$measured at $\sim 50 M$ in simulations of models $D 1$ with an initial pressure depletion of $2 \%$ (dotted line) and without any added perturbation (solid line). Right panel: magnification of the initial spurious burst.

the matter and those of the horizons during the collapse (not shown here) are very similar in both the perturbed and the unperturbed models.

A similar conclusion can be drawn when considering the gravitational-wave emission and this is summarized in figure 8, whose left panel shows the $Q_{20}^{+}$waveforms for model $D 1$ with the initial perturbation (dotted line, cf figure 5, centre-left panel) and without (solid line). Note that the two curves are not shifted in time and thus the delay is effectively due to the smaller initial amplitude of the unstable eigenmode when $\Delta p / p=0$. Note that not all of the signal coming from the unperturbed model is shifted in time and indeed also in this case 
an initial spurious burst of radiation is present between and 0 and $0.25 \mathrm{~ms}$, as highlighted in the right panel of figure 8. As mentioned earlier, this signal originates essentially from the truncation error introduced when interpolating onto a Cartesian grid the initial stellar models which are computed as equilibrium models in a code using spherical polar coordinates [47]. As a result, it is always present, with a form which is essentially independent of the stellar rotation rate (cf figure 5), but with an amplitude which can be further increased if the star is perturbed and hence with a larger initial violation of the constraint equations. This is very evident in the right panel of figure 8 which shows the two signals being well superposed in phase but also having different amplitudes, with the one coming from the unperturbed star being systematically smaller.

It is as yet uncertain whether this initial signal, albeit spurious, reflects a consistent response of the star to a perturbation and can therefore be associated with a $w$ mode [48]. Preliminary investigations in this direction seem to support the idea that the gravitational signal between 0 and $0.25 \mathrm{~ms}$ does indeed correspond to a $w$ mode (the signal does not converge away with resolution as shown in figure 4 of [31]) and could therefore be used to extract the eigenfrequencies of these modes in rapidly rotating stars which are yet unaccessible to perturbative studies. However, further work is needed to consolidate this conclusion.

We note that while introducing an initial small perturbation can serve to accelerate the matter dynamics, which however are not influenced noticeably, a large pressure perturbation can instead lead to significantly different results and even to incorrect interpretations on the efficiency of the gravitational-wave emission during the collapse. This is quite clear in figure 9, whose left panel shows the changes in the even-parity waveform $Q_{20}^{+}$emitted during the collapse of model $D 4$ when this is subject to pressure depletions going from 2 to $99 \%$. Clearly, as the pressure depletion is increased, the gravitational collapse becomes much more rapid, asymptotically becoming the one produced in the free-fall of an oblate distribution of dust. Also the corresponding changes in the mass quadrupole from this increasingly rapid collapse are larger and thus the amplitude of the gravitational-wave emission is increased as well. It is not surprising, therefore, that in these conditions it can easily reach values comparable with the ones computed in [3], where pressure reductions between 60 and $99 \%$ were indeed used.

In order to find a closer comparison with the values reported in [3] we show in the right panel of figure 9 the energy emitted in gravitational waves as a function of the pressure depletion for model $D 4$. Note, however, that in the case of large pressure depletions, the collapse is so rapid that it is very difficult, if possible at all, to distinguish the initial-burst signal from that produced by the collapse. Indeed, although the height of the first peak of the signal is closely related to the amplitude of the initial perturbation and grows monotonically with it, this is not true for the other peaks, as shown in the left panel of figure 9. As a result, while the open circles refer to the total signal, the filled ones instead show the emitted energy when the first peak in the signal is not taken into account and hence without the initial burst. It is evident that, as the pressure removal is increased, the energy radiated increases, becoming about two orders of magnitude larger than that obtained in the absence of perturbations. Such large values are in good agreement with those presented in [3] and induce us to conclude that the estimates made there, even if they served as useful upper limits, were dominated by the unrealistic dynamics of the matter.

Interestingly, the total energy emitted in gravitational radiation (when not including the initial burst) does not have a monotonic behaviour with $\Delta p / p$ and two different factors may combine to yield this effect. The first one is that, as the pressure support is drastically reduced, the centrifugal support, that in model $D 4$ plays an important dynamical role, ceases to be relevant and the dust-like matter collapses with only a small increase in the oblateness and 

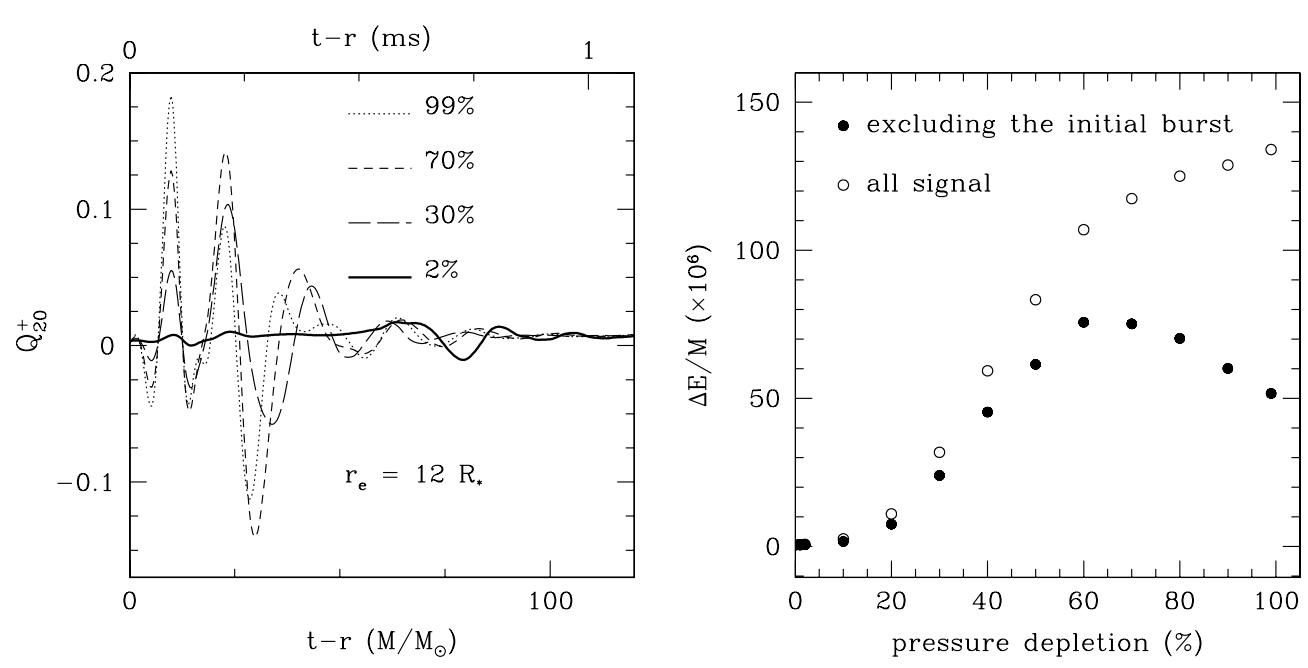

Figure 9. Left panel: comparison of $Q_{20}^{+}$from simulations of model $D 4$, differing in the amount of initial pressure depletion. Right panel: energy carried by the emitted gravitational waves during the collapse of model $D 4$ with different percentages of initial pressure depletion. All measurements were performed at a coordinate distance of $\sim 50 \mathrm{M}$.

hence in the mass quadrupole. The second factor is that in the more rapid collapse triggered by larger pressure depletions, the apparent horizon is produced much earlier and hence a larger amount of radiation remains trapped and cannot reach the observer. While of little practical interest because of the extreme conditions of matter involved, verifying these conjectures may provide important information on the behaviour of the Einstein equations in a nonlinear regime and deserves further investigations.

As a final remark, we note that an initial small perturbation also has an obvious drawback when it comes to analysing the gravitational-wave signal. The signal from the early burst of radiation, in fact, combines with the stronger collapse signal and can lead to incorrect estimates about the efficiency of the emission of gravitational radiation during the collapse for slowly rotating models. In the case of model $D 1$, for instance, the energy contained in the initial burst amounts to $\sim 30 \%$ of the energy produced instead during the actual gravitational collapse. For model $D 4$, on the other hand, this amounts only to $\sim 2 \%$.

\subsection{Perturbations in the velocity}

A pressure reduction is not the only possible perturbation that can be introduced in order to induce the collapse of a star past the secular stability limit. Another possibility, also used in the past in [49], consists in adding an inward-directed radial velocity to the equilibrium configuration, which we have here done in terms of a radial velocity of constant modulus 0.02 throughout the star.

This different type of initial perturbation gives rise to a slightly larger $(\lesssim 2 \%)$ violation of the constraint equations at the initial time and produces a collapse over a timescale which is comparable to that resulting from a $10 \%$ depletion of the pressure support. The efficiency in the energy emission, on the other hand, can be much larger as it will be discussed in more detail in the following section. 

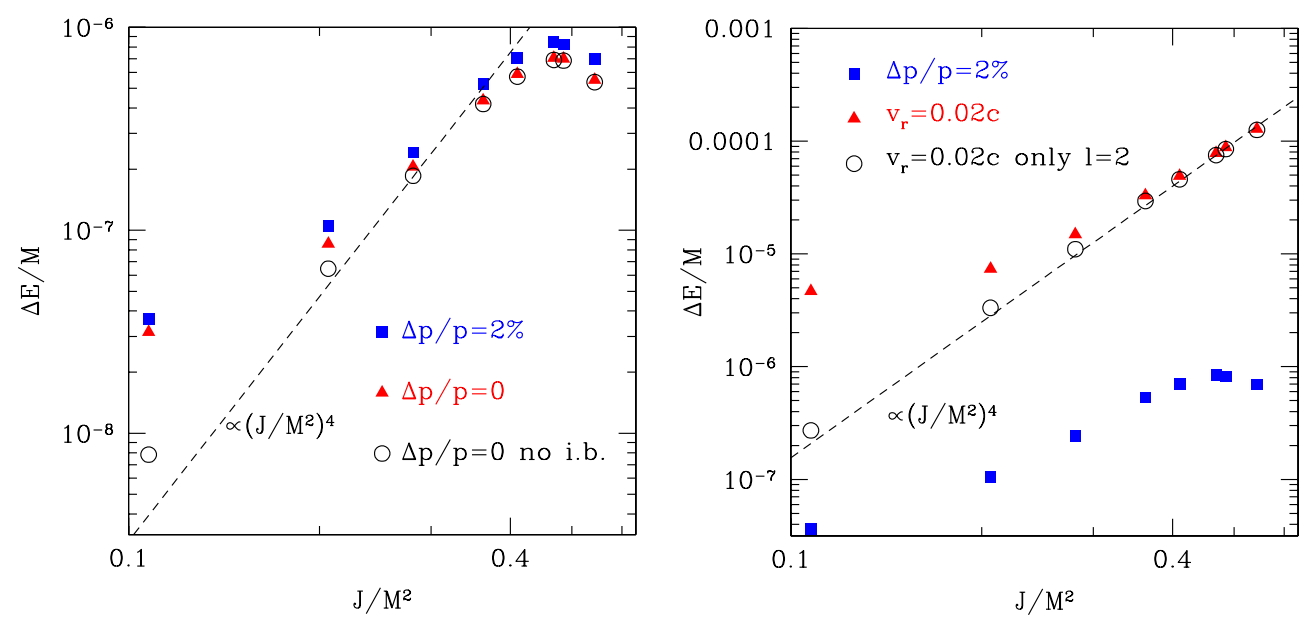

Figure 10. Energy carried by the emitted gravitational waves during the collapse for different values of the rotation parameter $J / M^{2}$ and initial perturbations. Left panel: filled squares and triangles refer respectively to models with a $2 \%$ pressure perturbation and to models that are unperturbed. Open circles refer to the same as the filled symbols but after excluding the initial burst in the waveforms (see section 6.2). Right panel: filled triangles refer to models perturbed with an inward uniform radial velocity of 0.02 and the open circles to the same models but considering only the $\ell=2$ contribution to the energy; filled squares refer again to pressure-perturbed models and are used as a reference. In both panels the measurements are made at a coordinate distance of $50 M$ and the dashed lines indicate a scaling $\propto\left(J / M^{2}\right)^{4}$.

(This figure is in colour only in the electronic version)

\section{Energy efficiency and detectability}

Determining the energy efficiency in the emission of gravitational radiation in fully nonlinear regimes of the Einstein equations is particularly difficult as perturbative or post-Newtonian approaches cannot be used reliably. The role that numerical-relativity calculations can play in this context is therefore particularly valuable and it represents one of the goals of most simulations. In addition, determining this efficiency in the case of the gravitational collapse to a black hole is made more difficult by the intrinsic weakness of the system which loses only a small fraction of its binding energy to gravitational radiation. In the case of binary black hole calculations, in contrast, the efficiency can easily reach a few per cent even in the simplest scenario of non-spinning, equal-mass binaries.

In figure 10 we present a summary of the efficiency in the collapse to black hole by reporting in a log-log plot the emitted energy as a function of the initial stellar rotation rate parameter $J / M^{2}$ and for different initial perturbations. A discussion on how to calculate this energy from the gauge-invariant quantities can be found in [31, 45] and it has been here calculated for an observer at a coordinate distance of $50 \mathrm{M}$. The left panel of figure 10, in particular, highlights the influence of pressure perturbations and shows with filled squares and triangles models with a $2 \%$ pressure perturbation and unperturbed models, respectively. Open circles, on the other hand, are the same as the filled symbols but when the initial burst in the waveforms is excluded (see section 6.2 for a discussion).

Clearly, and as first pointed out in [3], the efficiency follows behaviour of the type $\Delta E / E \propto\left(J / M^{2}\right)^{4}$ almost up to the largest rotation rates that yield equilibrium models in uniform rotation, i.e. $J / M^{2} \lesssim 0.54$. After that, the efficiency does not grow further and this represents a difference with respect to what found in [3], where the efficiency essentially 
saturated at very large rotation rates. (We recall that the rather crude way of introducing rotation in the initial models allowed values as large as $J / M^{2} \simeq 0.9$ to be reached in [3].) As mentioned in section 4 , this is probably due to the increased centrifugal support that these models experience and that effectively slows down the growth time for the dynamical instability (cf the right panel of figure 2). The value of $J / M^{2}$ at which the maximum efficiency is reached depends on the rapidity of the collapse and hence on the initial perturbation. For models with a small or zero initial perturbation, the maximum is located at $J / M^{2} \simeq 0.4$, while for more rapid collapses (as those shown in the right panel of figure 10), this happens at higher rotation rates. Note also that the efficiency does not follow a power-law behaviour at very small values of $J / M^{2}$. A comparison with the efficiency calculated not including the initial burst (open circles) shows that this is just the result of the initial spurious gravitational wave signal that, as mentioned above, can represent a significant fraction of the whole signal at low rotation rates.

The right panel of figure 10 also highlights the influence on the energy efficiency of velocity perturbations, with the filled triangles referring to models perturbed with an inward uniform radial velocity of 0.02 and with the open circles referring to the same models but when considering only the $\ell=2$ contribution to the energy. Clearly, velocity perturbations do not alter the overall scaling with rotation but do produce a significant increase in the efficiency, which can easily become two orders of magnitude larger than that produced with pressure perturbations (this is shown with filled squares as a reference). This enhanced emission is essentially the result of a more rapid change in mass quadrupole (indeed the amplitude of the $\ell=2$ mode is always larger than the corresponding mode in the cases of pressure depletion), but it also receives a contribution from higher-order multipoles, especially from the $\ell=4$ and at low rotation rates ( $\mathrm{cf}$ filled triangles and open circles in the right panel of figure 10). The evidence that the $\ell=4$ contribution to the overall energy is rather similar at all rotation rates seems to indicate that this is just an artefact of the initial perturbation and that a very clear scaling $\propto\left(J / M^{2}\right)^{4}$ is recovered when considering the $\ell=2$ contribution only (open circles). This result, on the other hand, also highlights that the study of the multipolar structure of the gravitational-wave emission from the collapse can be used to deduce the dynamical and kinematical properties of the star at the time of the collapse.

The gravitational-wave information computed here can also be used to determine the detectability of these sources [31, 45]. In the case of an interferometric detector with the sensitivity of Virgo and of the signal coming only from the gravitational collapse, we set an upper limit for the characteristic amplitude produced in the collapse of a rapidly and uniformlyrotating polytropic star at $10 \mathrm{kpc}$ to be $h_{c}=5.77 \times 10^{-22}\left(M / M_{\odot}\right)$ at a characteristic frequency $f_{c}=931 \mathrm{~Hz}$. In the case of a detector with the sensitivity of LIGO I, instead, we obtain $h_{c}=5.46 \times 10^{-22}\left(M / M_{\odot}\right)$ at $f_{c}=531 \mathrm{~Hz}$. The resulting signal-to-noise ratios are then $(S / N)_{\mathrm{D} 1-\mathrm{D} 4}^{\mathrm{Virgo}} \simeq 0.27-2.1,(S / N)_{\mathrm{D} 1-\mathrm{D} 4}^{\text {advL }} \simeq 1.2-11$ and $(S / N)_{\mathrm{D} 1-\mathrm{D} 4} \simeq 3.3-28$ for detectors such as Virgo/LIGO, advanced LIGO or Dual [50].

\section{Conclusions}

We have provided details and presented additional results on the numerical study of the gravitational-wave emission from the collapse of neutron stars to rotating black holes in three dimensions [1, 2]. In particular, we have discussed the advantages and disadvantages of the use of the excision technique and how alternative approaches to that of excision can be used with great success to extract the complete gravitational-wave signal [2].

As a first step towards the characterization of these sources of gravitational waves, we have presented a systematic discussion of the influence that rotation and different perturbations 
have on the waveforms and hence on the energy emitted in gravitational waves. In particular, a systematic analysis of the waveforms calculated under different initial rotation rates has provided the first estimates in full general relativity and for rapidly rotating stars of the growth time for the dynamical instability to axisymmetric perturbations and confirmed the existence of a precise power-law scaling of the energy efficiency in terms of the rotation parameter $J / M^{2}$.

We have also shown that the pressure perturbations traditionally used to trigger the collapse do not affect sensitively the dynamics of the matter and of the trapped surfaces as long as they are very small. Excessively large pressure depletions, on the other hand, can change significantly the way the collapse proceeds as well as artificially amplify the energy efficiency in the emission of gravitational waves. This clarifies the source of the differences between our estimates for the efficiency and those made in axisymmetry in [3]. Furthermore, the study of the waveforms produced with perturbations of different amplitudes and type has also made it possible to isolate the part of the signal produced by the actual collapse from the spurious one which should instead be related to initial violations of the constraint equations and which is produced either from the interpolation of the initial data onto a Cartesian grid or from the introduction of the initial perturbations. While it is still unclear whether this initial signal reflects a consistent response of the star to a perturbation and can therefore be associated with a $w$ mode, a number of considerations seem to support this hypothesis.

Overall, the results found indicate that the gravitational collapse of axisymmetric neutron stars to rotating black holes is not an efficient process for converting the binding energy into gravitational waves, with an overall efficiency $\delta M / M \simeq 10^{-7}-10^{-6}$ for uniformly rotating models. This efficiency, however, can be increased up to two orders of magnitude if velocity perturbations are present in the collapsing star and it is possible that similar conclusions may be valid also for the collapse of differentially rotating models.

As a concluding remark we note that, while this work, together with the ones preceding it $[1,2,8]$, has provided a full and consistent picture of the gravitational-wave emission from the collapse of neutron stars to rotating black holes, it represents only a very idealized description of this process. Additional and considerable work is still needed both in the modelling of the matter (through improved equations of state, the inclusion of the contributions coming from magnetic fields, radiation transport, multifluids, a solid crust, etc) and in the numerical techniques needed to handle this improved modelling. Both aspects will represent the focus of our future research.

\section{Acknowledgments}

It is a pleasure to thank Erik Schnetter for his help with the mesh refinement and for many useful discussions, and Nikolaos Stergioulas for help with the initial data. The computations presented here were performed on the Albert cluster at the University of Parma and the Peyote cluster at the Albert-Einstein-Institut.

\section{References}

[1] Baiotti L, Hawke I, Rezzolla L and Schnetter E 2005 Phys. Rev. Lett. 94131101 (Preprint gr-qc/0503016)

[2] Baiotti L and Rezzolla L 2006 Phys. Rev. Lett. 97141101

[3] Stark R F and Piran T 1985 Phys. Rev. Lett. 55891

[4] Baiotti L, Hawke I, Montero P and Rezzolla L 2003 Computational Astrophysics in Italy: Methods and Tools vol 1, ed R Capuzzo-Dolcetta (Trieste: Mem. Soc. Astron. It. Suppl.) p 210

[5] Goodale T, Allen G, Lanfermann G, Massó J, Radke T, Seidel E and Shalf J 2003 Vector and Parallel Processing_VECPAR'2002: 5th Int. Conf. (Lecture Notes in Computer Science) (Berlin: Springer)

[6] Cactus Computational Toolkit home page URL http://www.cactuscode.org/ 
[7] Alcubierre M, Brügmann B, Dramlitsch T, Font J A, Papadopoulos P, Seidel E, Stergioulas N and Takahashi R 2000 Phys. Rev. D 62044034 (Preprint gr-qc/0003071)

[8] Baiotti L, Hawke I, Montero P J, Löffler F, Rezzolla L, Stergioulas N, Font J A and Seidel E 2005 Phys. Rev. D 71024035 (Preprint gr-qc/0403029)

[9] Arnowitt R, Deser S and Misner C W 1962 Gravitation: An Introduction to Current Research ed L Witten (New York: Wiley) pp 227-65 (Preprint gr-qc/0405109)

[10] Nakamura T, Oohara K and Kojima Y 1987 Prog. Theor. Phys. Suppl. 90 1-218

[11] Shibata M and Nakamura T 1995 Phys. Rev. D 525428

[12] Baumgarte T W and Shapiro S L 1999 Phys. Rev. D 59024007 (Preprint gr-qc/9810065)

[13] Alcubierre M, Brügmann B, Diener P, Koppitz M, Pollney D, Seidel E and Takahashi R 2003 Phys. Rev. D 67084023 (Preprint gr-qc/0206072)

[14] Sarbach O, Calabrese G, Pullin J and Tiglio M 2002 Phys. Rev. D 66064002 (Preprint gr-qc/0205064)

[15] Bona C, Ledvinka T, Palenzuela C and Zacek M 2004 Phys. Rev. D 69064036 (Preprint gr-qc/0307067)

[16] Nagy G, Ortiz O E and Reula O A 2004 Phys. Rev. D 70044012 (Preprint gr-qc/0402123)

[17] Alcubierre M, Brügmann B, Pollney D, Seidel E and Takahashi R 2001 Phys. Rev. D 64 061501(R) (Preprint gr-qc/0104020)

[18] Bona C, Massó J, Seidel E and Stela J 1995 Phys. Rev. Lett. 75 600-3 (Preprint gr-qc/9412071)

[19] Alcubierre M 1997 Phys. Rev. D 55 5981-91 (Preprint gr-qc/9609015)

[20] Alcubierre M and Massó J 1998 Phys. Rev. D 57 R4511-5 (Preprint gr-qc/9709024)

[21] Smarr L and York J W 1978 Phys. Rev. D 17 2529-52

[22] Martí J M, Ibáñez J M and Miralles J M 1991 Phys. Rev. D 433794

[23] Banyuls F, Font J A, Ibáñez J M, Martí J M and Miralles J A 1997 Astrophys. J. 476221

[24] Ibáñez J, Aloy M, Font J, Martí J, Miralles J and Pons J 2001 Godunov Methods: Theory and Applications ed E Toro (New York: Kluwer/Plenum)

[25] Font J A 2003 Living Rev. Relativity 64 (URL http://www.livingreviews.org/Articles/lrr-2003-4)

[26] Martí J M and Müller E 1999 Living Rev. Relativity 23 (Preprint astro-ph/9906333) URL http://www. livingreviews.org/lrr-1999-3

[27] Rezzolla L and Zanotti O 2001 J. Fluid Mech. 449395

[28] Rezzolla L, Zanotti O and Pons J A 2003 J. Fluid Mech. 479199

[29] Colella P and Woodward P R 1984 J. Comput. Phys. 54174

[30] Schnetter E, Hawley S H and Hawke I 2004 Class. Quantum Grav. 21 1465-88 (Preprint gr-qc/0310042)

[31] Baiotti L, Hawke I, Rezzolla L and Schnetter E 2007 29th Spanish Relativity Meeting (ERE 2006): Einstein's Legacy: from the Theoretical Paradise to Astrophysical Observations (Palma de Mallorea, Spain) ed A S Olives and J Carot at press

[32] Berger M J and Oliger J 1984 J. Comput. Phys. 53 484-512

[33] Mesh Refinement with Carpet URL http://www.carpetcode.org/

[34] Kreiss H O and Oliger J 1973 Methods for the Approximate Solution of Time Dependent Problems vol 10 (Geneva: World Meteorological Organization, International Council of Scientic Unions)

[35] Friedman J L, Ipser J R and Sorkin R D 1988 Astrophys. J. 325 722-4

[36] Shibata M, Baumgarte T W and Shapiro S L 2000 Phys. Rev. D 61044012 (Preprint gr-qc/9911308)

[37] Ashtekar A, Beetle C and Fairhurst S 2000 Class. Quantum Grav. 17 253-98 (Preprint gr-qc/9907068)

[38] Ashtekar A, Beetle C, Dreyer O, Fairhurst S, Krishnan B, Lewandowski J and Wisniewski J 2000 Phys. Rev. Lett. 85 3564-7 (Preprint gr-qc/0006006)

[39] Ashtekar A, Beetle C and Lewandowski J 2001 Phys. Rev. D 64044016 (Preprint gr-qc/0103026)

[40] Ashtekar A and Krishnan B 2002 Phys. Rev. Lett. 89261101 (Preprint gr-qc/0207080)

[41] Dreyer O, Krishnan B, Shoemaker D and Schnetter E 2003 Phys. Rev. D 67024018 (Preprint gr-qc/0206008)

[42] Shibata M 2003 Phys. Rev. D 67024033 (Preprint gr-qc/0301103)

[43] Duez M D, Liu Y T, Shapiro S L and Stephens B C 2004 Phys. Rev. D 69104030 (Preprint gr-qc/0402502)

[44] Rezzolla L, Abrahams A M, Matzner R A, Rupright M E and Shapiro S L 1999 Phys. Rev. D 59064001 (Preprint gr-qc/9807047)

[45] Nagar A and Rezzolla L 2005 Class. Quantum Grav. 22 R167-92 (URL http://stacks.iop.org/0264-9381/22/ R167)

[46] Berti E, Cardoso V and Will C M 2006 Phys. Rev. D 73064030 (Preprint gr-qc/0512160)

[47] Stergioulas N and Friedman J L 1995 Astrophys. J. 444306

[48] Kokkotas K D and Schmidt B G 1999 Living Rev. Rel. 22 (http://www.livingreviews.org/lrr-1999-2)

[49] Bonazzola S, Frieben J, Gourgoulhon E and Marck J A 1996 Proc. 3rd Int. Conf. on Spectral and High Order Methods, Houston Journal of Mathematics (1996), University of Houston (Preprint gr-qc/9604029)

[50] Bonaldi M, Cerdonio M, Conti L, Pinard M, Prodi G A, Taffarello L and Zendri J P 2003 Phys. Rev. D 68102004 